\title{
A stochastic eco-epidemiological system with patchy structure and transport-related infection
}

\author{
Zhihui Ma ${ }^{1}$. Shuyan Han ${ }^{1}$. Shenghua $\mathrm{Li}^{1}$ \\ Received: 16 September 2020 / Revised: 26 July 2021 / Accepted: 21 October 2021 / \\ Published online: 13 November 2021 \\ ๑) The Author(s), under exclusive licence to Springer-Verlag GmbH Germany, part of Springer Nature 2021
}

\begin{abstract}
In this paper, a stochastic eco-epidemiological system with patchy structure and transport-related infection is proposed and the stochastic dynamical behaviors are investigated. Firstly, by constructing suitable Lyapunov functions, it is revealed that there is a unique globally positive solution starting from the positive initial value. Secondly, it is proved that the presented system is stochastically ultimately bounded and the average in time of the second moment of solution is bounded. Thirdly, we prove that the large enough stochastic perturbations may lead the predator population and the diseases in the predator to be extinct while it is persistent in the deterministic system. Finally, some numerical simulations are given to test our theoretical results.
\end{abstract}

Keywords Eco-epidemiological system · Patchy structure · Transport-related infection - Stochastic perturbation

AMS Subject Classification 37C75 - 34F05 - 92B05 · 92D30 - 93E03

\section{Introduction}

Eco-epidemiology is an important branch in mathematical biology which both considers the ecological and epidemiological factors. The influence of diseases in the ecological system is an important issue from the mathematical and ecological perspectives. Many researchers presented eco-epidemiological systems and tended to explore ecological systems being subject to epidemiological aspects (Anderson and May 1986; Venturino 1993; Bhattacharyya and Mukhopadhyay 2010; Venturino 2002; Saha and Samanta 2019; Greenhalgh et al. 2020; Haque and Venturino 2007; Hethcote

$凶$ Zhihui Ma

mazhh@1zu.edu.cn

1 School of Mathematics and Statistics, Lanzhou University, Lanzhou, Gansu 730000, People's Republic of China 
2000; Mainul 2010; Gulland 1995; Hsieh and Hsiao 2008; Fenton and Rands 2006). Anderson and May (Anderson and May 1986) firstly proposed an eco-epidemiological system which incorporates diseases into the interacting populations. After that, many researchers proposed and investigated eco-epidemiological systems for the spread of diseases among interacting species and revealed the spread mechanisms of diseases based on their studies (Bhattacharyya and Mukhopadhyay 2010; Venturino 2002; Saha and Samanta 2019; Greenhalgh et al. 2020; Haque and Venturino 2007). Some works studied the spread of diseases in predator/prey population in Lotka-Volterra type predation systems (Bhattacharyya and Mukhopadhyay 2010; Venturino 2002; Saha and Samanta 2019; Mainul 2010; Saha and Samanta 2020; Saha et al. 2018; Mondal and Samanta 2021). Hsieh and Hsiao (2008) and Fenton and Rands (2006) proposed a predator-prey system with disease in both prey and predator populations and considered the system dynamical behaviors. The results showed that the dynamical behaviors of predation systems with diseases are more complex than those without diseases. In fact, a certain disease in predator populations is a very common phenomenon and the biologically relevant examples in ecosystems are found by Gulland (1995). For examples, rabies and Sarcoptes spp. in foxes (Vulpis vulpis) and coyotes (Canis latrans), where rabbits are prey populations. The Phocine Distemper Virus in both the common seal (Phoca vitulina) and the striped dolphin (Stenella coeruleoalba), Oto- strongylus circumlitis, and Uncinaria lucasi, which affect ringed seals (Phoca hispida) and northern fur seals (Callorhinus ursinus), respectively. In this case, fishes are prey populations. The other example is that common seals (Phoca vitulina) and striped dolphins (Stenella coeruleoaba) are infected with canine distemper virus (PDV). In 1988, PDV infectious disease was found in seals. In 1988, PDV was first identified as the cause of death of 18,000 harbor seals (P. vitulina) and 300 gray seals (Halichoerus grypus) on the Nordic coast. In this case, fish is considered as prey and the disease will not cross the species barrier. The Avian Pox, Newcastle Disease, Influenza, Pasteurella multocida, Apergillus fumigatus and Leukocytozoon simondi are infectious agents and all affect a wide range of avian species, in which prey individuals are mainly insects (Venturino 2002).

On the one hand, the environment habitats are heterogeneous and mainly formed by a set of discrete patches. This induces populations survive in patchy habitats and are associated with their migrations. The migration rate explicates the evolutionarily stable patterns of the populations, and moreover plays an important role in persistence of the interacting populations. One of the popular way is the classical idea of evolutionary ecology where the less fit phenotypes, which are distinguished based on their migration and/or dispersal properties, are replaced by fitter ones as a result of evolution. Based on this issue, the ideal free distribution (IFD) as one of the popular concept are presented (Saha and Samanta 2019). Hence, the population systems in patchy habitats with migration (dispersion) are more realistic. However, it is inevitable that some infectious diseases may spread form one region to another as populations migrate or transport between regions. The migration or transportation among regions was one of the main factors which affected the outbreak of infectious diseases. The migration or transportation will change the disease's dynamics and induce infection diseases to be endemic even if infectious diseases will go to extinction in each region without transport-related infection. This will induce more serious consequences in 
population survival and evolution. Such as the influenza and COVID-19 will transmit form one country/city to other countries/cities. Therefore, the patchy model systems with the infectious diseases during transport are more realistic and essential, and have been studied by many researchers (Hamilton and May 1977; Abrams et al. 2007; Saha and Samanta 2019; Liu and Takeuchi 2006; Allen 2011; Cui et al. 2006; Allen and Bokil 2012; Hu et al. 2019; Liu et al. 2018; Rudnicki 2003; Ji and Jiang 2011; Liu et al. 2018; Bao and Shao 2015). For example, Liu and Takeuchi (2006) proposed an $S I Q S$ system to study the effect of transport-related infection and entry screening. The results revealed that the entry screening may be helpful for disease eradication since it can always have the possibility to eradicate the disease led by transport-related infection, and furthermore have the possibility to eradicate disease even when the disease is endemic in both isolated cities. Cui et al. (2006) presented an SI S epidemic model system to understand the effect of transport-related infection on the disease's spread and the corresponding mechanism. The results showed that the transport-related infection intensifies diseases spread if infectious diseases break out to cause an endemic situation in each region. However, almost all published researches for eco-epidemiological systems do not consider the possibility for individuals which may be infected during their travels. This factor plays a very important role in determining the spread and development of infectious diseases, and it is very essential to strengthen restrictions of passengers once infectious diseases appear. Motivated by this, this work firstly proposed an eco-epidemiological system incorporating the transport-related infection.

On the other hand, the dynamical behaviors of ecological populations are always affected by environmental noises or disturbances (Cui et al. 2006; Allen and Bokil 2012; Hu et al. 2019; Liu et al. 2018; Rudnicki 2003; Ji and Jiang 2011; Liu et al. 2018; Bao and Shao 2015; Mao 2007; Rebelo and Soresina 2020; Xin and Liu 2019; Arifah and Mao 2004; Li and Mao 2009; Das and Samanta 2018). Deterministic population models in ecology do not usually incorporate environmental fluctuation. They are often justified by the implicit assumption that stochastic deviations are small enough to be ignored in large populations. Deterministic models will be proved ecologically useful only if the dynamical patterns are still in evidence when stochastic effects are introduced. Uncertain changes of populations are usually considered as an effect of environmental stochasticity. In fact, the physical and biological environment of populations is not totally predictable, such as the population reproduction depends on temperature, humidity, parasites, pathogens, environmental pollution and so all. Hence, the population's development and growth should be considered as a stochastic process rather than a deterministic one. Rudnicki (2003) provided a detailed analysis for stability of a stochastic Lotka-Volterra system. Ji and Jiang (2011) and Liu et al. (2018) studied a predator-prey system with modified Leslie-Gower and Holling type II schemes with stochastic perturbations. Bao and Shao (2015) further extended this system into a stochastic predation system with all coefficients exhibiting random fluctuations. The published researches have revealed that the environmental fluctuations will induce the birth rate, death rate, transmission coefficient and other parameters of dynamical systems to exhibit randomness with a greater or lesser extent. Moreover, the random fluctuations could apply to control some infectious diseases. Motivated 
by this, this paper proposed an eco-epidemiological system incorporating the patchy structure, transport-related inflection and stochastic perturbation.

The paper is organized as follows. In Sect. 2, an stochastic eco-epidemiological system with patchy structure and transport-related infection is proposed. The unique global positivity of solutions of the presented system is given in Sect. 3. Section 4 shows that the presented model system is stochastically ultimately bounded and the average in time of the second moment of solution is bounded. In Sect. 5, we will deduce the condition of extinction of the total predator populations and disease in the predators. Finally, the theoretical results are discussed and some numerical simulations are given to test our theoretical results.

\section{System description}

Based on the above realistic issues, suppose that diseases only spread in predator population, and predator population is divided into two classes: the susceptible predator and the infected predator. Let $X_{i}, S_{i}$ and $I_{i}$ represent the number of prey, susceptible predator, infected predator in patch $i(i=1,2)$, respectively.

The basic assumptions for system formation are as follows:

(i) In the absence of predators, the prey population grows logistically with $r_{i}\left(1-\frac{X_{i}}{K_{i}}\right)$ in patch $i(i=1,2)$, respectively.

(ii) Holling I functional response is adopted for susceptible predators, and they will lose the predation ability after being infected by diseases.

(iii) The standard incidence rate is adopted in the spread of diseases among predator populations, and $\frac{\beta_{i} S_{i} I_{i}}{S_{i}+I_{i}}$ is the total number of new cases per unit time in patch $i(i=1,2)$, respectively. However, this paper supposes that the mass-action incidence rate for the infection during transports due to the sufficient and frequent contacts between susceptible and infected predators, that is, $\gamma_{i} \alpha_{i j}^{2} S_{i} I_{i}$ is the total number of new cases per unit time during transport from patch $i$ to patch $j$, $(i, j=1,2, i \neq j)$.

(iv) The birth rate and death rate of predator individuals who are traveling are omitted since the birth and death processes are more fast than the predators' one-way transport between two patches.

(v) It is assumed that stochastic perturbations are white noises which are directly proportional to $X_{i}(t), S_{i}(t)$ and $I_{i}(t)(i=1,2)$, and influenced on the $\dot{X}_{i}(t)$, $\dot{S}_{i}(t)$ and $\dot{I}_{i}(t)$ in the proposed system $(i=1,2)$ respectively. Hence, the random perturbations incorporate into incidence functions in patch 1 and patch 2 are as follows:

$$
\frac{\beta_{1} S_{1} I_{1}}{S_{1}+I_{1}} d t \rightarrow \frac{\left(\beta_{1}+\eta_{1} \dot{B}_{4}\right) S_{1} I_{1}}{S_{1}+I_{1}} d t, \quad \frac{\beta_{2} S_{2} I_{2}}{S_{2}+I_{2}} d t \rightarrow \frac{\left(\beta_{2}+\eta_{2} \dot{B}_{8}\right) S_{2} I_{2}}{S_{2}+I_{2}} d t,
$$

Based on the above assumptions and diagram, the following compartment diagram is presented: 


\section{Prey}

Patch 1:

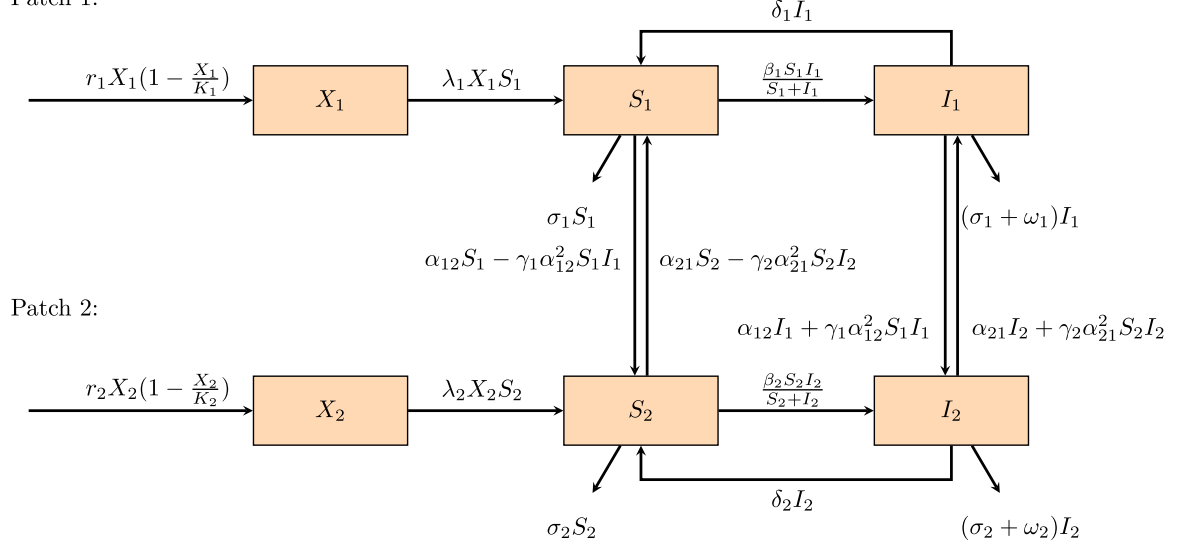

Fig. 1 Compartment diagram

Based on the above assumptions and diagram, the following stochastic ecoepidemiological system with patchy structure and transport-related infection is proposed:

$$
\left\{\begin{aligned}
d X_{1}(t) & =\left[r_{1} X_{1}\left(1-\frac{X_{1}}{K_{1}}\right)-\lambda_{1} X_{1} S_{1}\right] d t+a_{1} X_{1} d B_{1}(t), \\
d S_{1}(t) & =\left[p_{1} X_{1} S_{1}-\frac{\beta_{1} S_{1} I_{1}}{S_{1}+I_{1}}-\sigma_{1} S_{1}+\delta_{1} I_{1}-\alpha_{12} S_{1}+\alpha_{21} S_{2}-\gamma_{2} \alpha_{21}^{2} S_{2} I_{2}\right] d t \\
& +b_{1} S_{1} d B_{2}(t)-\frac{\eta_{1} S_{1} I_{1}}{S_{1}+I_{1}} d B_{4}(t), \\
d I_{1}(t) & =\left[\frac{\beta_{1} S_{1} I_{1}}{S_{1}+I_{1}}-\left(\sigma_{1}+\omega_{1}\right) I_{1}-\delta_{1} I_{1}-\alpha_{12} I_{1}+\alpha_{21} I_{2}+\gamma_{2} \alpha_{21}^{2} S_{2} I_{2}\right] d t \\
& +c_{1} I_{1} d B_{3}(t)+\frac{\eta_{1} S_{1} I_{1}}{S_{1}+I_{1}} d B_{4}(t), \\
d X_{2}(t) & =\left[r_{2} X_{2}\left(1-\frac{X_{2}}{K_{2}}\right)-\lambda_{2} X_{2} S_{2}\right] d t+a_{2} X_{2} d B_{5}(t), \\
d S_{2}(t) & =\left[p_{2} X_{2} S_{2}-\frac{\beta_{2} S_{2} I_{2}}{S_{2}+I_{2}}-\sigma_{2} S_{2}+\delta_{2} I_{2}-\alpha_{21} S_{2}+\alpha_{12} S_{1}-\gamma_{1} \alpha_{12}^{2} S_{1} I_{1}\right] d t \\
& +b_{2} S_{2} d B_{6}(t)-\frac{\eta_{2} S_{2} I_{2}}{S_{2}+I_{2}} d B_{8}(t), \\
d I_{2}(t) & =\left[\frac{\beta_{2} S_{2} I_{2}}{S_{2}+I_{2}}-\left(\sigma_{2}+\omega_{2}\right) I_{2}-\delta_{2} I_{2}-\alpha_{21} I_{2}+\alpha_{12} I_{1}+\gamma_{1} \alpha_{12}^{2} S_{1} I_{1}\right] d t \\
& +c_{2} I_{2} d B_{7}(t)+\frac{\eta_{2} S_{2} I_{2}}{S_{2}+I_{2}} d B_{8}(t) .
\end{aligned}\right.
$$

The ecological and epidemiological meanings of all parameters of system (2) are given in Table 1 , where $r_{i}, K_{i}, \lambda_{i}, p_{i}, \beta_{i}, \sigma_{i}, \omega_{i}, \alpha_{i j}, \delta_{i}, \gamma_{i},(i, j=1,2, i \neq j)$ are all positive. $B_{i}(t)$ are independent standard Brownian motions with $B_{i}(0)=0$ and $a_{j}^{2}, b_{j}^{2}, c_{j}^{2}, \eta_{j}^{2}, \xi_{j}^{2}$ are all positive and denote the intensities of the white noises $(i=1,2,3,4,5,6,7,8, j=1,2)$.

From the biological point of view, the term $\alpha_{i j} S_{i}$ means that the susceptible predators leave the patch $i$ and $\gamma_{i} \alpha_{i j}^{2} S_{i} I_{i}$ denotes the susceptible predators become infected 
Table 1 Definition of the parameters in system (2.1)

\begin{tabular}{ll}
\hline Parameter & Definitions \\
\hline$r_{i}$ & Intrinsic birth rate of the prey species at patch $i$ \\
$\lambda_{i}$ & Predation rate at patch $i$ \\
$K_{i}$ & Carrying capacity of prey species at patch $i$ \\
$p_{i}$ & Biomass conversion rate at patch $i$ \\
$\beta_{i}$ & The effective contact rate at patch $i$ \\
$\sigma_{i}$ & Natural death rate of the predator at patch $i$ \\
$\delta_{i}$ & The individuals' rate of recovery due to natural causes or treatment \\
$\omega_{i}$ & Disease-induced death rate of the predator at patch $i$ \\
$\alpha_{i j}$ & The travel rate from patch $i$ to patch $j$ \\
$\gamma_{i}$ & The effective contact rate in the transportation from patch $i$ to patch $j$ \\
\hline
\end{tabular}

during their travels from patch $i$ to patch $j$. Therefore, it is assumed that the term $\alpha_{i j} S_{i}-\gamma_{i} \alpha_{i j}^{2} S_{i} I_{i}$ should be nonnegative and the term $1-\gamma_{i} \alpha_{i j} I_{i}$ is positive throughout this paper.

Defining $\phi(t)=\left(X_{1}(t), S_{1}(t), I_{1}(t), X_{2}(t), S_{2}(t), I_{2}(t)\right)$, and the initial conditions of system (2.1) are as follow:

$$
\begin{gathered}
\phi(0)=\left(X_{1}(0), S_{1}(0), I_{1}(0), X_{2}(0), S_{2}(0), I_{2}(0)\right), X_{i}(0)>0, \\
S_{i}(0)>0, I_{i}(0)>0, i=1,2 .
\end{gathered}
$$

Let $\left(\Omega, \mathcal{F},\left\{\mathcal{F}_{t}\right\}_{t \geq 0}, \mathbb{P}\right)$ be a complete probability space with a filtration $\left\{\mathcal{F}_{t}\right\}_{t \geq 0}$ satisfying the usual condition (i.e., it is increasing and right continuous while $\overline{\mathcal{F}}_{0}$ contains all $\mathbb{P}$-null sets), $B_{i}(t)(i=1,2,3,4,5,6,7,8)$ are defined on this complete probability space. Meanwhile, defining $\mathbb{R}_{+}^{d}=\left\{x \in \mathbb{R}^{d}: x_{i}>0,1 \leq i \leq d\right\}$.

Generally, we consider the following $d$-dimensional stochastic differential equation:

$$
d x(t)=f(x(t), t) d t+g(x(t), t) d B(t),
$$

with initial value $x(0)=x_{0} \in \mathbb{R}^{d}$. Where $f(x(t), t)$ is a function defined on $\mathbb{R}^{d} \times$ $\left[t_{0},+\infty\right), g(x(t), t)$ is a $d \times m$ matrix, $f, g$ are locally Lipschitz continuous in $x$, $B(t)$ denotes an $m$-dimensional standard Brownian motion defined on the complete probability space $\left(\Omega, \mathcal{F},\left\{\mathcal{F}_{t}\right\}_{t \geq 0}, \mathbb{P}\right)$. The differential operator $L$ of system (2.1) is defined by

$$
L=\frac{\partial}{\partial t}+\sum_{i=1}^{d} f_{i}(t) \frac{\partial}{\partial x_{i}}+\frac{1}{2} \sum_{i, j=1}^{d}\left[g^{T}(t, x) g(t, x)\right]_{i j} \frac{\partial^{2}}{\partial x_{i} \partial x_{j}}
$$


If $L$ acts on a function $V \in C^{2,1}\left(\mathbb{R}^{d} \times\left[t_{0},+\infty\right] ; \mathbb{R}_{+}\right)$, then

$$
L V(x, t)=V_{t}(x, t)+V_{x}(x, t) f(x, t)+\frac{1}{2} \operatorname{trace}\left[g^{T}(x, t) V_{x x} g(x, t)\right],
$$

where $V_{t}=\frac{\partial V}{\partial t}, V_{x}=\left(\frac{\partial V}{\partial x_{1}}, \ldots, \frac{\partial V}{\partial x_{d}}\right), V_{x x}=\left(\frac{\partial^{2} V}{\partial x_{i} \partial x_{i}}\right)_{d \times d}, C^{2,1}\left(\mathbb{R}^{d} \times\left[t_{0},+\infty\right]\right.$; $\mathbb{R}_{+}$) denote the family of all nonnegative functions defined on $\mathbb{R}^{d} \times\left[t_{0},+\infty\right)$ and continuously twice differentiable in $x$ and once in $t$. By Itós formula, if $x(t) \in \mathbb{R}^{d}$, we have

$$
d V(x, t)=L V(x, t) d t+V_{x}(x, t) g(x, t) d B(t) .
$$

Definition 2.1 (Xin and Liu 2019) System (2.1) is said to be ultimately bounded in mean if there is a positive constant $L$ independent of initial conditions (2.2) such that

$$
\limsup _{t \rightarrow \infty} \mathbb{E}|\phi(t)| \leq L
$$

Definition 2.2 (Arifah and Mao 2004; Li and Mao 2009). The solution $\phi(t)$ of system (2.1) is said to be stochastically ultimately bounded, if for any $\epsilon \in(0,1)$, there is a positive constant $B=B(\epsilon)$, such that for any initial value (2.2), the solution $\phi(t)$ of system (2.1) has the property that

$$
\limsup _{t \rightarrow \infty} \mathbb{P}\{|\phi(t)|>B\} \leq \epsilon
$$

\section{Existence and uniqueness of the global positive solution}

In this section, we will prove that system (2.1) has a unique global positive solution by using Lyapunov analysis method.

Theorem 3.1 For any given initial conditions (2.2), there is a unique positive solution $\phi(t)$ of system $(2.1)$ on $[0, \infty)$, and the solution $\phi(t)$ will remain in $\mathbb{R}_{+}^{6}$ with probability one.

Proof Since the coefficients of system (2.1) are locally Lipschitz continuous, for any given initial value (2.2), there is a unique local solution in $\left[0, \tau_{e}\right)$, where $\tau_{e}$ denotes the explosion time.

Now, we will prove that the solution is global, i.e. $\tau_{e}=+\infty$ a.s. To this end, let $k_{0} \geq 1$ be sufficiently large such that $X_{i}(0) \in\left(\frac{1}{k_{0}}, k_{0}\right), S_{i}(0) \in\left(\frac{1}{k_{0}}, k_{0}\right), I_{i}(0) \in$ $\left(\frac{1}{k_{0}}, k_{0}\right), i=1,2$. For each integer $k \geq k_{0}$ define the stopping time as follow

$$
\tau_{k}=\inf \left\{t \in\left[0, \tau_{e}\right): X_{i}(t) \notin\left(\frac{1}{k}, k\right) \text { or } S_{i}(t) \notin\left(\frac{1}{k}, k\right) \text { or } I_{i}(t) \notin\left(\frac{1}{k}, k\right), i=1,2\right\},
$$


we denote as the empty set and set inf $=\infty$ throughout this paper. Clearly, $\tau_{k}$ is increasing as $k \rightarrow \infty$. Set $\tau_{\infty}=\lim _{k \rightarrow \infty} \tau_{k}$, hence $\tau_{\infty} \leq \tau_{e}$ a.s. If we can show that $\tau_{\infty}=\infty$ a.s., then $\tau_{e}=\infty$ and $\phi(t) \in \mathbb{R}_{+}^{6}$ a.s. for all $t \geq 0$. If this assertion is not true, then there exist $T>0$ and $\epsilon \in(0,1)$ such that $\mathbb{P}\left\{\tau_{\infty} \leq T\right\}>\epsilon$. For any $k \geq k_{0}$, let $\Omega_{k}=\left\{\omega \in \Omega: \tau_{k}(\omega) \leq T\right\}$. That is, $\mathbb{P}\left(\Omega_{k}\right)>\epsilon$ for any $k \geq k_{0}$.

Define the $\mathbb{C}^{6}$-function $\mathrm{V}: \mathbb{R}_{+}^{6} \rightarrow \mathbb{R}_{+}$by

$$
V(\phi(t))=\sum_{i=1}^{2}\left(2 X_{i}-1-\ln X_{i}+2 S_{i}-1-\ln S_{i}+2 I_{i}-1-\ln I_{i}\right)
$$

For any $0 \leq t<\left(\tau_{k} \wedge T\right)$, using Itố $\hat{o}^{\prime}$ formula, we have

$$
\begin{aligned}
d V= & L V d t+\left(2-\frac{1}{X_{1}}\right) a_{1} X_{1}(t) d B_{1}(t)+\left(2-\frac{1}{S_{1}}\right) b_{1} S_{1}(t) d B_{2}(t)+\left(2-\frac{1}{I_{1}}\right) c_{1} I_{1}(t) d B_{3}(t) \\
& +\frac{\eta_{1}\left(I_{1}-S_{1}\right)}{S_{1}+I_{1}} d B_{4}(t)+\left(2-\frac{1}{X_{2}}\right) a_{2} X_{2}(t) d B_{5}(t)+\left(2-\frac{1}{S_{2}}\right) b_{2} S_{2}(t) d B_{6}(t) \\
& +\left(2-\frac{1}{I_{2}}\right) c_{2} I_{2}(t) d B_{7}(t)+\frac{\eta_{2}\left(I_{2}-S_{2}\right)}{S_{2}+I_{2}} d B_{8}(t),
\end{aligned}
$$

where

$$
\begin{aligned}
L V= & \sum_{i, j=1, i \neq j}^{2}\left\{\left(2-\frac{1}{X_{i}}\right)\left[r_{i} X_{i}\left(1-\frac{X_{i}}{K_{i}}\right)-\lambda_{i} X_{i} S_{i}\right]\right. \\
& +\left(2-\frac{1}{S_{i}}\right)\left[p_{i} X_{i} S_{i}-\frac{\beta_{i} S_{i} I_{i}}{S_{i}+I_{i}}-\sigma_{i} S_{i}+\delta_{i} I_{i}\right. \\
& \left.-\alpha_{i j} S_{i}+\alpha_{j i} S_{j}-\gamma_{j} \alpha_{j i}^{2} S_{j} I_{j}\right]+\left(2-\frac{1}{I_{i}}\right) \\
& {\left[\frac{\beta_{i} S_{i} I_{i}}{S_{i}+I_{i}}-\left(\sigma_{i}+\omega_{i}\right) I_{i}-\delta_{i} I_{i}-\alpha_{i j} I_{i}+\alpha_{j i} I_{j}\right.} \\
& \left.\left.+\gamma_{j} \alpha_{j i}^{2} S_{j} I_{j}\right]+\frac{1}{2}\left[a_{i}^{2}+b_{i}^{2}+\frac{\eta_{i}^{2} I_{i}^{2}}{\left(S_{i}+I_{i}\right)^{2}}+c_{i}^{2}+\frac{\eta_{i}^{2} S_{i}^{2}}{\left(S_{i}+I_{i}\right)^{2}}\right]\right\} \\
\leq & \sum_{i, j=1, i \neq j}^{2}\left\{\frac{\left(2 K_{i} r_{i}+r_{i}-K_{i} p_{i}\right)^{2}}{8 K_{i} r_{i}}-r_{i}+2 \sigma_{i}+\delta_{i}+\omega_{i}\right. \\
& \left.+2 \alpha_{i j}+\beta_{i}+\eta_{i}^{2}+\frac{1}{2}\left(a_{i}^{2}+b_{i}^{2}+c_{i}^{2}\right)+\lambda_{i} S_{i}\right\} .
\end{aligned}
$$

Using the inequality $u \leq 2 u-1-\ln u$ for any $u \in \mathbb{R}_{+}$, we have

$$
L V \leq G+\max \left\{\lambda_{1}, \lambda_{2}\right\} V
$$

where $G=\sum_{i, j=1, i \neq j}^{2}\left\{\frac{\left(2 K_{i} r_{i}+r_{i}-K_{i} p_{i}\right)^{2}}{8 K_{i} r_{i}}-r_{i}+2 \sigma_{i}+\delta_{i}+\omega_{i}+2 \alpha_{i j}+\beta_{i}+\eta_{i}^{2}+\right.$ $\left.\frac{1}{2}\left(a_{i}^{2}+b_{i}^{2}+c_{i}^{2}\right)\right\}$ is a suitable constant which is independent of $X_{i}, S_{i}, I_{i}$ and $t$. 
Furthermore, for any $k>k_{0}$, integrating both sides of (3.1) from 0 to $\tau_{k} \wedge T$, and then take the expectation, we get

$$
\begin{aligned}
\mathbb{E} \mathbb{V}\left(\phi\left(\tau_{k} \wedge T\right)\right) & \leq V(\phi(0))+G \mathbb{E}\left(\tau_{k} \wedge T\right)+\max \left\{\lambda_{1}, \lambda_{2}\right\} \int_{0}^{\tau_{k} \wedge T} \mathbb{E} \mathbb{V}(\phi(t) d t \\
& \leq V(\phi(0))+G T+\max \left\{\lambda_{1}, \lambda_{2}\right\} \int_{0}^{\tau_{k} \wedge T} \mathbb{E} \mathbb{V}(\phi(t)) d t
\end{aligned}
$$

By the Gronwall inequality (Liu et al. 2018), it is obtained that

$$
\left.\mathbb{E} \mathbb{V}\left(\phi\left(\tau_{k} \wedge T\right)\right) \leq(\phi(0))+G T\right) e^{\max \left\{\lambda_{1}, \lambda_{2}\right\} T}
$$

Noting that for every $\omega \in \Omega_{k}$, there is at least one of $X_{1}\left(\tau_{k}, \omega\right), S_{1}\left(\tau_{k}, \omega\right), I_{1}\left(\tau_{k}, \omega\right)$, $X_{2}\left(\tau_{k}, \omega\right), S_{2}\left(\tau_{k}, \omega\right)$ or $I_{2}\left(\tau_{k}, \omega\right)$ that is equal to $k$ or $\frac{1}{k}$. Hence, $V\left(\phi\left(\tau_{k}, \omega\right)\right)$ is no less than either $2 k-1-\ln k$ or $\frac{2}{k}-1-\ln \frac{1}{k}=\frac{2}{k}-1+\ln k$, i.e

$$
V\left(\phi\left(\tau_{k}, \omega\right)\right) \geq(2 k-1-\ln k) \wedge\left(\frac{2}{k}-1+\ln k\right) .
$$

It follows from (3.2) and (3.3), we have

$$
\begin{aligned}
(V(\phi(0))+G T) e^{\max \left\{\lambda_{1}, \lambda_{2}\right\} T} & \geq \mathbb{E} \mathbb{V}\left(\phi\left(\tau_{k} \wedge T\right)\right) \\
& \geq \mathbb{E}\left[\mathbb{I}_{\Omega_{k}(\omega)} V\left(\phi\left(\tau_{k}, \omega\right)\right)\right] \\
& \geq \epsilon_{1}(2 k-1-\ln k) \wedge\left(\frac{2}{k}-1+\ln k\right)
\end{aligned}
$$

where $\mathbb{I}_{\Omega_{k}(\omega)}$ denotes the indicator function of $\Omega_{k}$. Let $k \rightarrow+\infty$, then

$$
+\infty>(V(\phi(0))+G T) e^{\max \left\{\lambda_{1}, \lambda_{2}\right\} T}=+\infty,
$$

which leads to a contradiction. Hence, it must have $\tau_{\infty}=+\infty$ a.s. as required.

Next, we claim that $\phi(t)$ is positive almost surely on $[0, \infty)$. On $t \in[0, \infty)$, for the system (2.1), we have

$$
X_{i}(t)=X_{i}(0) e^{\int_{0}^{t}\left(r_{i}\left(1-\frac{X_{i}(\theta)}{K_{i}}\right)-\lambda_{i} S_{i}(\theta)\right) d \theta+a_{i} B_{k}(t)}
$$

where $i=1, k=1, i=2, k=4$.

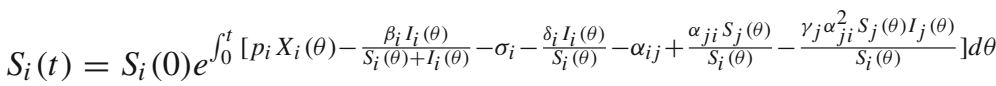

$$
\begin{aligned}
& +b_{i} B_{k}(t)-\int_{0}^{t} \frac{\eta_{i} I_{i}(\theta)}{S_{i}(\theta)+I_{i}(\theta)} d B_{m}(\theta),
\end{aligned}
$$


where $i, j=1,2, i \neq j, k=2,6, m=4,8$.

$$
I_{i}(t)=I_{i}(0) e^{\int_{0}^{t}\left(\frac{\beta_{i} S_{i}(\theta)}{S_{i}(\theta)+I_{i}(\theta)}-\left(\sigma_{i}+\omega_{i}+\delta_{i}+\alpha_{i j}\right)+\frac{\alpha_{j i} I_{j}(\theta)}{I_{i}(\theta)}+\frac{\gamma_{j} \alpha_{j i}^{2} S_{j}(\theta) I_{j}(\theta)}{I_{i}(\theta)}\right) d \theta+c_{i} B_{k}(t)+\int_{0}^{t} \frac{\eta_{i} S_{i}(\theta)}{S_{i}(\theta)+I_{i}(\theta)} d B_{m}(\theta)},
$$

where $i, j=1,2, i \neq j, k=3,7, m=4,8$.

Apparently, for the initial condition (2.2), we have $X_{i}(0)>0, S_{i}(0)>0, I_{i}(0)>0$, then $X_{i}(t)>0, S_{i}(t)>0, I_{i}(t)>0, i=1,2$.

Therefore, (2.1) with (2.2) has the unique global solution $\phi(t)$ on $t \in[0, \infty)$ and the solution will remain in $\mathbb{R}_{+}^{6}$ with probability one.

This completes the proof.

\section{Stochastically ultimate boundedness}

In this section, we examine the ultimate boundedness in mean, stochastically ultimate boundedness, and the average in time of the second moment of solution.

Theorem 4.1 For any given initial values (2.2), solution $\phi(t)$ of system (2.1) satisfies

$$
\limsup _{t \rightarrow \infty} \mathbb{E}|\phi(t)| \leq \frac{K_{1}\left(r_{1}+\xi\right)^{2}}{4 r_{1} \xi}+\frac{K_{2}\left(r_{2}+\xi\right)^{2}}{4 r_{2} \xi}
$$

where $\xi=\min \left\{\sigma_{1}, \sigma_{2}\right\}$, Further, system (2.1) is ultimately bounded in mean.

Proof By Theorem 3.1, the solution $\phi(t)$ will remain in $\mathbb{R}_{+}^{6}$ for all $t>0$ with probability one. Define the $\mathbb{C}^{6}$-function $\mathrm{V}: \mathbb{R}_{+}^{6} \rightarrow \mathbb{R}$ by

$$
V_{1}(t, \phi(t))=e^{\xi t}\left(X_{1}+X_{2}+S_{1}+S_{2}+I_{1}+I_{2}\right)
$$

Applying Itố's formula, we have

$$
\begin{aligned}
& d V_{1}(t, \phi(t))=L V_{1}(t, \phi(t))+e^{\xi t}\left(a_{1} X_{1} d B_{1}+b_{1} S_{1} d B_{2}+c_{1} I_{1} d B_{3}\right. \\
& \left.\quad+a_{2} X_{2} d B_{5}+b_{2} S_{2} d B_{6}+c_{2} I_{2} d B_{7}\right),
\end{aligned}
$$

where

$$
\begin{aligned}
L V_{1}(t, \phi(t)) & =e^{\xi t} \sum_{i=1}^{2}\left\{-\frac{r_{i}}{K_{i}} X_{i}^{2}+\left(r_{i}+\xi\right) X_{i}-\left(\lambda_{i}-p_{i}\right) X_{i} S_{i}\right. \\
& \left.-\left(\sigma_{i}-\xi\right) S_{i}-\left(\sigma_{i}+\omega_{i}-\xi\right) I_{i}\right\} \\
& \leq e^{\xi t}\left[\frac{K_{1}\left(r_{1}+\xi\right)^{2}}{4 r_{1}}+\frac{K_{2}\left(r_{2}+\xi\right)^{2}}{4 r_{2}}\right] .
\end{aligned}
$$


Integrating both sides of (4.1) from 0 to $t$ and using the above equality, we obtain

$$
\begin{aligned}
V_{1}(t, \phi(t)) \leq & V_{1}(0, \phi(0))+\left[\frac{K_{1}\left(r_{1}+\xi\right)^{2}}{4 r_{1}}+\frac{K_{2}\left(r_{2}+\xi\right)^{2}}{4 r_{2}}\right] \int_{0}^{t} e^{\xi s} d s \\
& +\int_{0}^{t} e^{\xi s}\left[a_{1} X_{1}(s) d B_{1}(s)+b_{1} S_{1}(s) d B_{2}(s)+c_{1} I_{1}(s) d B_{3}(s)\right] \\
& +\int_{0}^{t} e^{\xi s}\left[a_{2} X_{2}(s) d B_{5}(s)+b_{2} S_{2}(s) d B_{6}(s)+c_{2} I_{2}(s) d B_{7}(s)\right] .
\end{aligned}
$$

Now, multiplying both sides of (4.2) by $e^{-\xi t}$, and taking the expectation, we have

$$
\begin{aligned}
\mathbb{E}\left[X_{1}(t)+X_{2}(t)+S_{1}(t)+S_{2}(t)+I_{1}(t)+I_{2}(t)\right] \leq & e^{-\xi t} V_{1}(0, \phi(0))+\left[\frac{K_{1}\left(r_{1}+\xi\right)^{2}}{4 r_{1}}\right. \\
& \left.+\frac{K_{2}\left(r_{2}+\xi\right)^{2}}{4 r_{2}}\right] \frac{1}{\xi}\left(1-e^{-\xi t}\right) .
\end{aligned}
$$

Furthermore, letting $t \rightarrow \infty$, we get

$$
\begin{aligned}
& \limsup _{t \rightarrow \infty} \mathbb{E}\left[X_{1}(t)+X_{2}(t)+S_{1}(t)+S_{2}(t)+I_{1}(t)+I_{2}(t)\right] \leq \frac{K_{1}\left(r_{1}+\xi\right)^{2}}{4 r_{1} \xi} \\
& \quad+\frac{K_{2}\left(r_{2}+\xi\right)^{2}}{4 r_{2} \xi} .
\end{aligned}
$$

Noting from the equality $|\phi(t)| \leq X_{1}(t)+X_{2}(t)+S_{1}(t)+S_{2}(t)+I_{1}(t)+I_{2}(t)$, for any $\phi(t) \in \mathbb{R}^{6}$, hence

$$
\lim \sup _{t \rightarrow \infty} \mathbb{E}|\phi(t)| \leq \frac{K_{1}\left(r_{1}+\xi\right)^{2}}{4 r_{1} \xi}+\frac{K_{2}\left(r_{2}+\xi\right)^{2}}{4 r_{2} \xi} .
$$

This completes the proof.

By Chebyshev's inequality and Theorem 4.1, we have the following conclusion.

Theorem 4.2 For any given initial values (2.2), the solution $\phi(t)$ of system (2.1) is stochastically ultimately bounded.

Theorem 4.3 Assume that there are positive numbers $m_{i}, n_{i}, l_{i}, \theta_{i}$, such that $R_{i}:=$ $2 \sigma_{i}+\left(2-m_{i}\right) \alpha_{i j}-m_{i} \alpha_{j i}-2 p_{i} K_{i}-n_{i} \delta_{i}-b_{i}^{2}-\frac{\eta_{i}^{2}}{2}>0, U_{i}:=2\left(\sigma_{i}+\omega_{i}\right)+(2-$ $\left.n_{i}\right) \delta_{i}-2 \beta_{i}+\left(2-l_{i}\right) \alpha_{i j}-l_{i} \alpha_{j i}-c_{i}^{2}-\frac{\eta_{i}^{2}}{2}-\theta_{i} \max \left\{\gamma_{1} \alpha_{12}^{2}, \gamma_{2} \alpha_{21}^{2}\right\} B>0, i=1,2$, where $m_{1} m_{2}=1, n_{1} n_{2}=1, l_{1} l_{2}=1, \theta_{1} \theta_{2}=1$. Then, for any given initial values (2.2), the solution $\phi(t)$ of system (2.1) satisfies

$$
\limsup _{t \rightarrow \infty} \frac{1}{t} \int_{0}^{t} \mathbb{E}|\phi(s)|^{2} d s \leq Q+K_{1}^{2}+K_{2}^{2},
$$


where $Q:=\frac{\sum_{i, j=1, i \neq j}^{2}\left\{2 \sigma_{i}+2 \alpha_{i j}+\beta_{i}+\omega_{i}+\delta_{i}+\frac{1}{2}\left(b_{i}^{2}+c_{i}^{2}+2 \eta_{i}^{2}\right)\right\}}{\min _{i, j=1,2, i \neq j}\left\{R_{i}, U_{i}\right\}}$ is a positive constant. That is to say, the average in time of the second moment of solution $\phi(t)$ is bounded.

Proof Firstly, Integrating both sides of $d\left(X_{1}(t)\right) \leq r_{1} X_{1}\left(1-\frac{X_{1}}{K_{1}}\right)+a_{1} X_{1} d B_{1}(t)$ from 0 to $t$ and taking the expectation, we have

$$
0 \leq \mathbb{E} \mathbb{X}_{1}(t) \leq X_{1}(0)+\mathbb{E} \int_{0}^{t}\left[-\frac{r_{1}}{K_{1}} X_{1}^{2}(s)+r_{1} X_{1}(s)\right] d s
$$

This implies

$$
\limsup _{t \rightarrow \infty} \frac{1}{t} \int_{0}^{t} \mathbb{E} \mathbb{X}_{1}^{2}(s) d s \leq K_{1}^{2}
$$

By the same calculation, we can obtain that

$$
\limsup _{t \rightarrow \infty} \frac{1}{t} \int_{0}^{t} \mathbb{E} \mathbb{X}_{2}^{2}(s) d s \leq K_{2}^{2}
$$

By theorem 4.1 and theorem 4.2, we have $S_{1}(t)+S_{2}(t)<B=\frac{K_{1}\left(r_{1}+\xi\right)^{2}}{4 r_{1} \xi \epsilon}+\frac{K_{2}\left(r_{2}+\xi\right)^{2}}{4 r_{2} \xi \epsilon}$ for any sufficiently small $\epsilon>0$.

Define the $\mathbb{C}^{6}$-function $\mathrm{V}: \mathbb{R}_{+}^{6} \rightarrow \mathbb{R}$ by

$$
V=\sum_{i=1}^{2}\left\{\left(S_{i}^{2}-\ln S_{i}+I_{i}^{2}-\ln I_{i}\right)\right\}
$$

Applying Itố $\hat{o}^{\prime}$ formula and the inequality $2 a b \leq a^{2}+b^{2}$, we have

$$
\begin{aligned}
d V \leq & \sum_{i, j=1, i \neq j}^{2}\left\{-\left[2 \sigma_{i}+\left(2-m_{i}\right) \alpha_{i j}-m_{i} \alpha_{j i}-2 p_{i} K_{i}-n_{i} \delta_{i}-b_{i}^{2}-\frac{\eta_{i}^{2}}{2}\right] S_{i}^{2}-\left[2\left(\sigma_{i}+\omega_{i}\right)\right.\right. \\
& \left.+\left(2-n_{i}\right) \delta_{i}-2 \beta_{i}+\left(2-l_{i}\right) \alpha_{i j}-l_{i} \alpha_{j i}-c_{i}^{2}-\frac{\eta_{i}^{2}}{2}-\theta_{i} \max \left\{\gamma_{1} \alpha_{12}^{2}, \gamma_{2} \alpha_{21}^{2}\right\} B\right] I_{i}^{2} \\
& \left.+2 \sigma_{i}+2 \alpha_{i j}+\beta_{i}+\omega_{i}+\delta_{i}+\frac{1}{2}\left(b_{i}^{2}+c_{i}^{2}+2 \eta_{i}^{2}\right)\right\} d t+\left(2 S_{1}-\frac{1}{S_{1}}\right) b_{1} S_{1} d B_{2}(t) \\
& +\left(2 I_{1}-\frac{1}{I_{1}}\right) c_{1} I_{1} d B_{3}(t)+\frac{\eta_{1}\left(I_{1}-S_{1}\right)\left(2 S_{1} I_{1}+1\right)}{S_{1}+I_{1}} d B_{4}(t)+\left(2 S_{2}-\frac{1}{S_{2}}\right) b_{2} S_{2} d B_{6}(t) \\
& +\left(2 I_{2}-\frac{1}{I_{2}}\right) c_{2} I_{2} d B_{7}(t)+\frac{\eta_{2}\left(I_{2}-S_{2}\right)\left(2 S_{2} I_{2}+1\right)}{S_{2}+I_{2}} d B_{8}(t) .
\end{aligned}
$$


Integrating both sides of the above inequality from 0 to $t$ and taking the expectation, we get

$$
\begin{aligned}
0 \leq & \mathbb{E} \mathbb{V}(\phi(t)) \leq V(\phi(0))+\sum_{i, j=1, i \neq j}^{2}\left\{\left[2 \sigma_{i}+2 \alpha_{i j}+\beta_{i}+\omega_{i}+\delta_{i}\right.\right. \\
& \left.+\frac{1}{2}\left(b_{i}^{2}+c_{i}^{2}+2 \eta_{i}^{2}\right)\right] t-\left[2 \sigma_{i}\right. \\
& \left.+\left(2-m_{i}\right) \alpha_{i j}-m_{i} \alpha_{j i}-2 p_{i} K_{i}-n_{i} \delta_{i}-b_{i}^{2}-\frac{\eta_{i}^{2}}{2}\right] \\
& \int_{0}^{t} \mathbb{E} \mathbb{S}_{i}^{2}(s) d s-\left[2\left(\sigma_{i}+\omega_{i}\right)+\left(2-n_{i}\right) \delta_{i}\right. \\
& \left.\left.-2 \beta_{i}+\left(2-l_{i}\right) \alpha_{i j}-l_{i} \alpha_{j i}-c_{i}^{2}-\frac{\eta_{i}^{2}}{2}-\theta_{i} \max \left\{\gamma_{1} \alpha_{12}^{2}, \gamma_{2} \alpha_{21}^{2}\right\} B\right] \int_{0}^{t} \mathbb{E} \mathbb{I}_{i}^{2}(s) d s\right\}
\end{aligned}
$$

It is straightforward to see that

$$
\limsup _{t \rightarrow \infty} \frac{1}{t} \int_{0}^{t} \mathbb{E}\left(S_{1}(s)^{2}+I_{1}(s)^{2}+S_{2}(s)^{2}+I_{2}(s)^{2}\right) d s \leq Q .
$$

Hence

$$
\limsup _{t \rightarrow \infty} \frac{1}{t} \int_{0}^{t} \mathbb{E}|\phi(s)|^{2} d s \leq Q+K_{1}^{2}+K_{2}^{2} .
$$

This completes the proof.

\section{Asymptotic pathwise estimation and extinction}

In this section, we consider asymptotic pathwise estimation the solution of system (2.1) and the extinction of the total predator and the extinction of ecological epidemics with probability one. Because the predator can travel freely between two patches, the extinction considered in the section must occur simultaneously in two patches. Firstly, we give the proof of the extinction of the total predator with probability one.

Theorem 5.1 For any given initial values (2.2), the solution $\phi(t)$ of system (2.1) satisfies

$$
\limsup _{t \rightarrow \infty} \frac{\ln \left(S_{1}(t)+I_{1}(t)+S_{2}(t)+I_{2}(t)\right)}{t} \leq H_{1} \text { a.s. }
$$

where $H_{1}$ is defined in (5.1). Moreover, if $H_{1}<0$ holds, then the total predator of system (2.1) will tend to zero exponentially with probability one.

Proof Let

$$
V_{2}(\phi(t))=\ln \left(S_{1}+I_{1}+S_{2}+I_{2}\right)
$$


Applying Itố's formula, we have

$$
d V_{2}=L V_{2} d t+\frac{1}{S_{1}+I_{1}+S_{2}+I_{2}}\left(b_{1} S_{1} d B_{2}(t)+c_{1} I_{1} d B_{3}(t)+b_{2} S_{2} d B_{6}(t)+c_{2} I_{2} d B_{7}(t)\right),
$$

where

$$
\begin{aligned}
L V_{2}= & \frac{1}{S_{1}+I_{1}+S_{2}+I_{2}}\left[p_{1} X_{1} S_{1}-\sigma_{1} S_{1}-\left(\sigma_{1}+\omega_{1}\right) I_{1}+p_{2} X_{2} S_{2}-\sigma_{2} S_{2}-\left(\sigma_{2}+\omega_{2}\right) I_{2}\right] \\
& -\frac{1}{2\left(S_{1}+I_{1}+S_{2}+I_{2}\right)^{2}}\left(b_{1}^{2} S_{1}^{2}+c_{1}^{2} I_{1}^{2}+b_{2}^{2} S_{2}^{2}+c_{2}^{2} I_{2}^{2}\right) \\
\leq & \left.\frac{1}{S_{1}+I_{1}+S_{2}+I_{2}}\left[p_{1} K_{1} S_{1}-\sigma_{1} S_{1}-\left(\sigma_{1}+\omega_{1}\right) I_{1}+p_{2} K_{2} S_{2}-\sigma_{2} S_{2}-\left(\sigma_{2}+\omega_{2}\right) I_{2}\right] .1\right) \\
& -\frac{1}{2} \min \left\{b_{1}^{2}, c_{1}^{2}, b_{2}^{2}, c_{2}^{2}\right\} \frac{S_{1}^{2}+I_{1}^{2}+S_{2}^{2}+I_{2}^{2}}{\left(S_{1}+I_{1}+S_{2}+I_{2}\right)^{2}} \\
\leq & -\min \left\{\sigma_{1}-p_{1} K_{1}, \sigma_{1}+\omega_{1}, \sigma_{2}-p_{2} K_{2}, \sigma_{2}+\omega_{2}\right\}-\frac{1}{8} \min \left\{b_{1}^{2}, c_{1}^{2}, b_{2}^{2}, c_{2}^{2}\right\} \\
:= & H_{1} .
\end{aligned}
$$

Hence,

$$
d V_{2} \leq H_{1} d t+b_{1} d B_{2}(t)+c_{1} d B_{3}(t)+b_{2} d B_{6}(t)+c_{2} d B_{7}(t)
$$

Integrating both sides of (5.2) from 0 to $t$ and then dividing $t$, we get

$$
\begin{aligned}
\frac{\ln \left(S_{1}(t)+I_{1}(t)+S_{2}(t)+I_{2}(t)\right)}{t} \leq & \frac{\ln \left(S_{1}(0)+I_{1}(0)+S_{2}(0)+I_{2}(0)\right)}{t} \\
& +H_{1}+\frac{b_{1} B_{2}(t)}{t}+\frac{c_{1} B_{3}(t)}{t}+\frac{b_{2} B_{6}(t)}{t}+\frac{c_{2} B_{7}(t)}{t} .
\end{aligned}
$$

By the Strong law of large numbers for martingales, we have $\lim _{t \rightarrow \infty} \frac{B_{i}(t)}{t}=0$, $i=2,3,6,7$.

Thus,

$$
\limsup _{t \rightarrow \infty} \frac{\ln \left(S_{1}(t)+I_{1}(t)+S_{2}(t)+I_{2}(t)\right)}{t} \leq H_{1} .
$$

It is straightforward to see that, when $H_{1}<0$ is satisfied, $S_{1}+I_{1}+S_{2}+I_{2}$ will go to zero exponentially with probability one, i.e the total predator of two patches will go to extinction simultaneously exponentially with probability one.

This completes the proof.

Remark 1 On the one hand, it is easy to see that $H_{1}=-\min \left\{\sigma_{1}-p_{1} K_{1}, \sigma_{1}+\omega_{1}, \sigma_{2}-\right.$ $\left.p_{2} K_{2}, \sigma_{2}+\omega_{2}\right\}-\frac{1}{8} \min \left\{b_{1}^{2}, c_{1}^{2}, b_{2}^{2}, c_{2}^{2}\right\}$ is decreasing with $b_{1}^{2}, c_{1}^{2}, b_{2}^{2}, c_{2}^{2}$ increasing. Hence, the total number of predators in two patches will die out exponentially as long as $b_{1}^{2}, c_{1}^{2}, b_{2}^{2}$ and $c_{2}^{2}$ are large enough such that $H_{1}<0$. This means that the white noise may lead predator population to be extinction while they are persistent in the 
deterministic system. On the other hand, we can obtain that $\limsup _{t \rightarrow \infty} \frac{\ln X_{i}(t)}{t} \leq r_{i}-\frac{a_{i}^{2}}{2}$ a.s. $(i=1,2)$. That is to say, if the white noise is large enough, the prey population will be extinct in statistic system while they are persistent in the deterministic system. Therefore, the larger white noise will have negative effects on the population coexistence.

Next, we will give the proof of the extinction of the disease with probability one by using the same method as Theorem 5.1 .

Theorem 5.2 For any given initial values (2.2), solution $\phi(t)$ of system (2.1) satisfies

$$
\limsup _{t \rightarrow \infty} \frac{\ln \left(I_{1}(t)+I_{2}(t)\right)}{t} \leq H_{2} \text { a.s. }
$$

where $\mathrm{H}_{2}$ is defined in (5.6). Moreover, if $\mathrm{H}_{2}<0$ holds, then the disease of system (2.1) will go to extinction exponentially with probability one.

Proof Define

$$
V_{3}(\phi(t))=\ln \left(I_{1}+I_{2}\right)
$$

Applying Itốs formula, we have

$$
\begin{aligned}
& d V_{3}=L V_{3} d t+\frac{1}{I_{1}+I_{2}}\left(c_{1} I_{1} d B_{3}(t)+\frac{\eta_{1} S_{1} I_{1}}{S_{1}+I_{1}} d B_{4}(t)+c_{2} I_{2} d B_{7}(t)\right. \\
& \left.+\frac{\eta_{2} S_{2} I_{2}}{S_{2}+I_{2}} d B_{8}(t)\right)
\end{aligned}
$$

where

$$
\begin{aligned}
L V_{3}= & \frac{1}{I_{1}+I_{2}}\left(\frac{\beta_{1} S_{1} I_{1}}{S_{1}+I_{1}}-\left(\sigma_{1}+\omega_{1}\right) I_{1}-\delta_{1} I_{1}+\gamma_{2} \alpha_{21}^{2} S_{2} I_{2}+\frac{\beta_{2} S_{2} I_{2}}{S_{2}+I_{2}}-\left(\sigma_{2}+\omega_{2}\right) I_{2}-\delta_{2} I_{2}\right. \\
& \left.+\gamma_{1} \alpha_{12}^{2} S_{1} I_{1}\right)-\frac{1}{2\left(I_{1}+I_{2}\right)^{2}}\left[c_{1}^{2} I_{1}^{2}+\frac{\eta_{1}^{2} S_{1}^{2} I_{1}^{2}}{\left(S_{1}+I_{1}\right)^{2}}+c_{2} I_{2}^{2}+\frac{\eta_{2}^{2} S_{2}^{2} I_{2}^{2}}{\left(S_{2}+I_{2}\right)^{2}}\right] \\
\leq & -\min \left\{\sigma_{1}+\omega_{1}+\delta_{1}, \sigma_{2}+\omega_{2}+\delta_{2}\right\}+\frac{1}{I_{1}+I_{2}}\left(\frac{\beta_{1} S_{1} I_{1}}{S_{1}+I_{1}}+\frac{\beta_{2} S_{2} I_{2}}{S_{2}+I_{2}}\right)+\frac{\gamma_{2} \alpha_{21}^{2} S_{2} I_{2}}{I_{1}+I_{2}} \\
& +\frac{\gamma_{1} \alpha_{12}^{2} S_{1} I_{1}}{I_{1}+I_{2}}-\frac{1}{2\left(I_{1}+I_{2}\right)^{2}}\left[\frac{\eta_{1}^{2} S_{1}^{2} I_{1}^{2}}{\left(S_{1}+I_{1}\right)^{2}}+\frac{\eta_{2}^{2} S_{2}^{2} I_{2}^{2}}{\left(S_{2}+I_{2}\right)^{2}}\right]-\frac{c_{1}^{2} I_{1}^{2}+c_{2} I_{2}^{2}}{2\left(I_{1}+I_{2}\right)^{2}} .
\end{aligned}
$$

Next, by theorem 4.1 and theorem 4.2 , we have $S_{1}(t)+S_{2}(t)<B=\frac{K_{1}\left(r_{1}+\xi\right)^{2}}{4 r_{1} \xi \epsilon}+$ $\frac{K_{2}\left(r_{2}+\xi\right)^{2}}{4 r_{2} \xi \epsilon}$ for any sufficiently small $\epsilon>0$.

Hence,

$L V_{3} \leq D+\frac{1}{I_{1}+I_{2}}\left(\frac{\beta_{1} S_{1} I_{1}}{S_{1}+I_{1}}+\frac{\beta_{2} S_{2} I_{2}}{S_{2}+I_{2}}\right)-\frac{1}{2\left(I_{1}+I_{2}\right)^{2}}\left[\frac{\eta_{1}^{2} S_{1}^{2} I_{1}^{2}}{\left(S_{1}+I_{1}\right)^{2}}+\frac{\eta_{2}^{2} S_{2}^{2} I_{2}^{2}}{\left(S_{2}+I_{2}\right)^{2}}\right]$,

where $D=-\min \left\{\sigma_{1}+\omega_{1}+\delta_{1}, \sigma_{2}+\omega_{2}+\delta_{2}\right\}-\frac{1}{4} \min \left\{c_{1}^{2}, c_{2}^{2}\right\}+\max \left\{\gamma_{2} \alpha_{21}^{2}, \gamma_{1} \alpha_{12}^{2}\right\} B$. 
Integrating both sides of (5.3) from 0 to $t$, we get

$$
\begin{aligned}
\ln \left(I_{1}(t)+I_{2}(t)\right) \leq & \ln \left(I_{1}(0)+I_{2}(0)\right)+D t+\int_{0}^{t} \frac{1}{I_{1}+I_{2}}\left(\frac{\beta_{1} S_{1} I_{1}}{S_{1}+I_{1}}+\frac{\beta_{2} S_{2} I_{2}}{S_{2}+I_{2}}\right) d u \\
& -\int_{0}^{t} \frac{1}{2\left(I_{1}+I_{2}\right)^{2}}\left[\frac{\eta_{1}^{2} S_{1}^{2} I_{1}^{2}}{\left(S_{1}+I_{1}\right)^{2}}+\frac{\eta_{2}^{2} S_{2}^{2} I_{2}^{2}}{\left(S_{2}+I_{2}\right)^{2}}\right] d u \\
& +c_{1} B_{3}(t)+c_{2} B_{7}(t)+N(t)
\end{aligned}
$$

where $N(t)=\int_{0}^{t} \frac{1}{I_{1}+I_{2}}\left[\frac{\eta_{1} S_{1} I_{1}}{S_{1}+I_{1}} d B_{4}(t)+\frac{\eta_{2} S_{2} I_{2}}{S_{2}+I_{2}} d B_{8}(t)\right]$ is a continuous local martingale whose quadratic variation is as follows

$$
\langle N, N\rangle_{t}=\int_{0}^{t} \frac{1}{\left(I_{1}+I_{2}\right)^{2}}\left[\frac{\eta_{1}^{2} S_{1}^{2} I_{1}^{2}}{\left(S_{1}+I_{1}\right)^{2}}+\frac{\eta_{2}^{2} S_{2}^{2} I_{2}^{2}}{\left(S_{2}+I_{2}\right)^{2}}\right] d u .
$$

By virtue of the exponential martingale inequality (Liu et al. 2018), it is obtained that

$$
P\left\{\sup _{0 \leq t \leq k}\left[N(t)-\frac{c}{2}\langle N, N\rangle_{t}\right]>\frac{2}{c} \ln k\right\} \leq \frac{1}{k^{2}},
$$

where $0<c<1, k$ is a random integer.

Applying Borel-Cantelli lemma (Liu et al. 2018) leads to that for almost all $\omega \in \Omega$, there exists a random integer $k_{0}>0$, such that for $k>k_{0}$, we have

$$
\sup _{0 \leq t \leq k}\left[N(t)-\frac{c}{2}\langle N, N\rangle_{t}\right] \leq \frac{2}{c} \ln k
$$

that is to say, for any $0 \leq t \leq k$, we get

$$
N(t) \leq \frac{c}{2} \int_{0}^{t} \frac{1}{\left(I_{1}+I_{2}\right)^{2}}\left[\frac{\eta_{1}^{2} S_{1}^{2} I_{1}^{2}}{\left(S_{1}+I_{1}\right)^{2}}+\frac{\eta_{2}^{2} S_{2}^{2} I_{2}^{2}}{\left(S_{2}+I_{2}\right)^{2}}\right] d u+\frac{2}{c} \ln k
$$

Substituting (5.5) into (5.4), we have

$$
\begin{aligned}
\ln \left(I_{1}(t)+I_{2}(t)\right) \leq & \ln \left(I_{1}(0)+I_{2}(0)\right)+D t+\int_{0}^{t} \frac{1}{I_{1}+I_{2}}\left(\frac{\beta_{1} S_{1} I_{1}}{S_{1}+I_{1}}+\frac{\beta_{2} S_{2} I_{2}}{S_{2}+I_{2}}\right) d u \\
& -\int_{0}^{t} \frac{1-c}{2\left(I_{1}+I_{2}\right)^{2}}\left[\frac{\eta_{1}^{2} S_{1}^{2} I_{1}^{2}}{\left(S_{1}+I_{1}\right)^{2}}+\frac{\eta_{2}^{2} S_{2}^{2} I_{2}^{2}}{\left(S_{2}+I_{2}\right)^{2}}\right] d u \\
& +c_{1} B_{3}(t)+c_{2} B_{7}(t)+\frac{2}{c} \ln k .
\end{aligned}
$$


Since

$$
\begin{aligned}
& \frac{\beta_{1} S_{1} I_{1}}{\left(S_{1}+I_{1}\right)\left(I_{1}+I_{2}\right)}-\frac{1-c}{2} \frac{\eta_{1}^{2} S_{1}^{2} I_{1}^{2}}{\left(S_{1}+I_{1}\right)^{2}\left(I_{1}+I_{2}\right)^{2}} \leq \frac{\beta_{1}^{2}}{2(1-c) \eta_{1}^{2}} \\
& \frac{\beta_{2} S_{2} I_{2}}{\left(S_{2}+I_{2}\right)\left(I_{1}+I_{2}\right)}-\frac{1-c}{2} \frac{\eta_{2}^{2} S_{2}^{2} I_{2}^{2}}{\left(S_{2}+I_{2}\right)^{2}\left(I_{1}+I_{2}\right)^{2}} \leq \frac{\beta_{2}^{2}}{2(1-c) \eta_{2}^{2}} .
\end{aligned}
$$

It is obtained that

$$
\begin{aligned}
\ln \left(I_{1}(t)+I_{2}(t)\right) \leq & \ln \left(I_{1}(0)+I_{2}(0)\right)+D t+c_{1} B_{3}(t)+c_{2} B_{7}(t) \\
& +\frac{2}{c} \ln k+\left[\frac{\beta_{1}^{2}}{2(1-c) \eta_{1}^{2}}+\frac{\beta_{2}^{2}}{2(1-c) \eta_{2}^{2}}\right] t
\end{aligned}
$$

Therefore, for $k-1<t<k$, one can see that

$$
\begin{aligned}
& \frac{\ln \left(I_{1}(t)+I_{2}(t)\right)}{t} \leq \frac{\ln \left(I_{1}(0)+I_{2}(0)\right)}{t}+c_{1} \frac{B_{3}(t)}{t}+c_{2} \frac{B_{7}(t)}{t}+\frac{2 \ln k}{c(k-1)} \\
& +D+\frac{\beta_{1}^{2}}{2(1-c) \eta_{1}^{2}}+\frac{\beta_{2}^{2}}{2(1-c) \eta_{2}^{2}} .
\end{aligned}
$$

Letting $k \rightarrow+\infty$, i.e., $t \rightarrow+\infty$ and in view of the Strong law of large numbers to the Brownian motion, we derive $\lim _{t \rightarrow \infty} \frac{B_{i}(t)}{t}=0, i=3,7$.

Consequently, we have

$$
\limsup _{t \rightarrow+\infty} \frac{\ln \left(I_{1}(t)+I_{2}(t)\right)}{t} \leq D+\frac{\beta_{1}^{2}}{2(1-c) \eta_{1}^{2}}+\frac{\beta_{2}^{2}}{2(1-c) \eta_{2}^{2}} .
$$

Letting $c \rightarrow 0$, we get

$$
\limsup _{t \rightarrow+\infty} \frac{\ln \left(I_{1}(t)+I_{2}(t)\right)}{t} \leq D+\frac{\beta_{1}^{2}}{2 \eta_{1}^{2}}+\frac{\beta_{2}^{2}}{2 \eta_{2}^{2}}:=H_{2} .
$$

It is straightforward to see that, when $H_{2}<0$ holds, $I_{1}+I_{2}$ will go to zero exponentially with probability one, i.e. the disease of two patches will go to extinction simultaneously exponentially with probability one.

This completes the proof.

Remark 2 It is easy to see that $H_{2}=-\min \left\{\sigma_{1}+\omega_{1}+\delta_{1}, \sigma_{2}+\omega_{2}+\delta_{2}\right\}-\frac{1}{4} \min \left\{c_{1}^{2}, c_{2}^{2}\right\}+$ $\min \left\{\gamma_{2} \alpha_{21}^{2}, \gamma_{1} \alpha_{12}^{2}\right\} B+\frac{\beta_{1}^{2}}{2 \eta_{1}^{2}}+\frac{\beta_{2}^{2}}{2 \eta_{2}^{2}}$ is decreasing in $c_{1}^{2}, c_{2}^{2}, \eta_{1}^{2}, \eta_{2}^{2}$. Hence, the disease in two patches will exponentially die out simultaneously as long as $c_{1}^{2}, c_{2}^{2}, \eta_{1}^{2}$ and $\eta_{2}^{2}$ are large enough such that $H_{2}<0$. This means that the white noise may lead diseases to be extinct while these diseases will be endemic in the deterministic system. Therefore, the white noise is beneficial to the infectious disease control. 
Table 2 Table of parameter values

\begin{tabular}{llllllllllllll}
\hline$r_{1}$ & $K_{1}$ & $\lambda_{1}$ & $p_{1}$ & $\beta_{1}$ & $\sigma_{1}$ & $\omega_{1}$ & $\delta_{1}$ & $\gamma_{1}$ & $\alpha_{12}$ & $a_{1}$ & $b_{1}$ & $c_{1}$ & $\eta_{1}$ \\
\hline 2 & 3.8 & 1 & 0.3 & 0.2 & 1.14 & 0.84 & 0.2 & 0.2 & 0.5 & 0.2 & 0.7 & 0.15 & 0.35 \\
$r_{2}$ & $K_{2}$ & $\lambda_{2}$ & $p_{2}$ & $\beta_{2}$ & $\sigma_{2}$ & $\omega_{2}$ & $\delta_{2}$ & $\gamma_{2}$ & $\alpha_{21}$ & $a_{2}$ & $b_{2}$ & $c_{2}$ & $\eta_{2}$ \\
1.5 & 2 & 1 & 0.5 & 0.3 & 1.5 & 1.24 & 0.15 & 0.3 & 0.63 & 0.3 & 0.65 & 0.4 & 0.2 \\
\hline
\end{tabular}

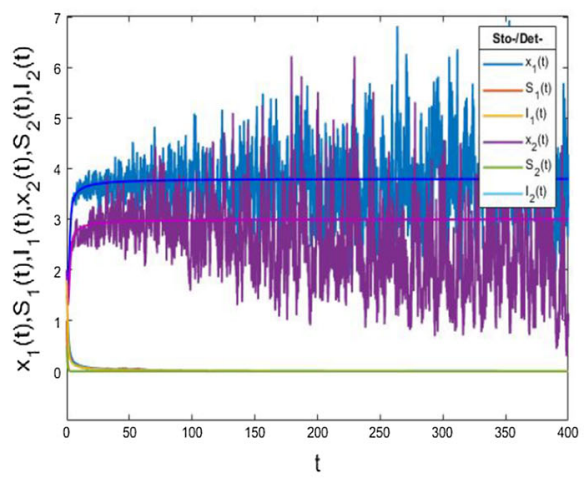

(a)

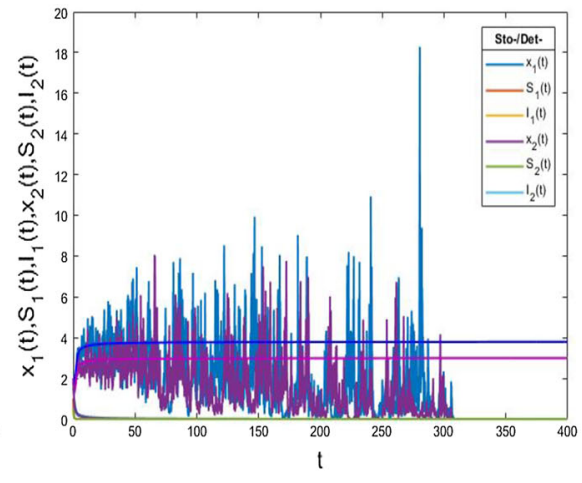

(b)

Fig. 2 The influence of the white noises when only the prey is present in the system (2.1), where a: $a_{1}=0.2, a_{2}=0.3, \mathbf{b}: a_{1}=2.2, a_{2}=2$

\section{Numerical results}

In this section, some numerical simulations for system (2.1) are conducted to illustrate our analytical results. The system parameter values are shown in Table 2 and we take initial values $\phi(0)=(2.5,1.5,0.5,2.5,1.5,0.5)$. Some of these parameter values have been taken from literatures (Saha and Samanta 2019).

In order to investigate the influence of stochastic perturbations on system (2.1), the numerical simulations on the stochastic system and the corresponding deterministic system under the same parameters are compared.

From Fig. 2a shows $r_{1}-\frac{a_{1}^{2}}{2}=1.98>0$ and $r_{2}-\frac{1}{a_{2}^{2}}=1.455>0$, and reveals that the relatively small white noise make the solutions of system (2.1) fluctuating in a large scale. Figure $2 b$ shows $r_{1}-\frac{a_{1}^{2}}{2}=-0.42<0$ and $r_{2}-\frac{1}{a_{2}^{2}}=-0.5<0$, and means that large enough white noise will lead to the extinction of prey population while it is persistent in the deterministic system .

Choosing $\sigma_{1}=0.56$ and $\sigma_{2}=0.51$ for Fig. 3, and the other parameters are the same as those in Table 2. Figure 3a shows $H_{1}=0.9871875>0$, and reveals that the relatively small white noise make the solutions of system (2.1) fluctuating in a large scale, while Fig. $3 b$ illustrates that the large enough white noise will lead to extinction of the predator population while it is persistent in the deterministic system because of $H_{1}=-0.5853125<0$, which is consistent with the theoretical results of Theorem 5.1 . 


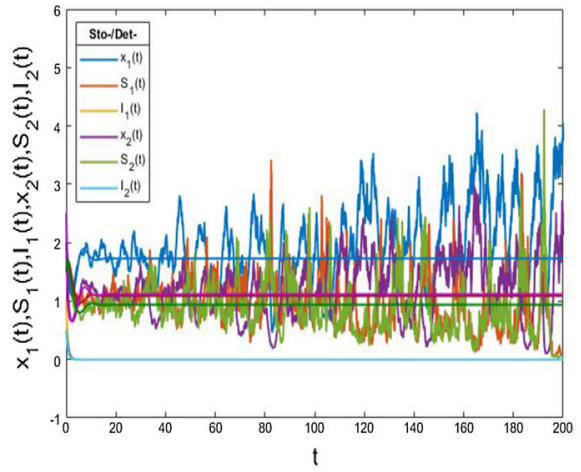

(a)

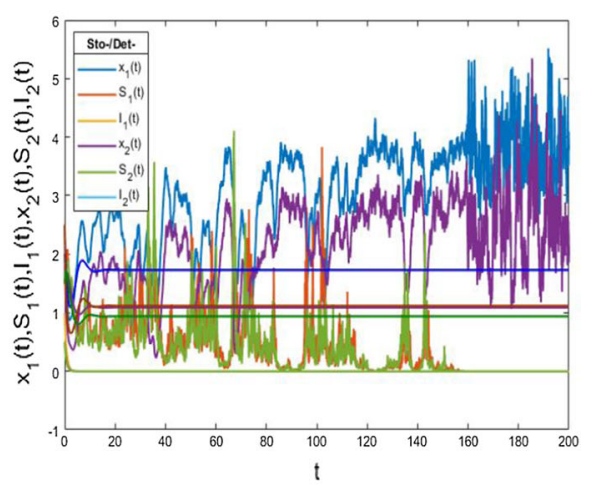

(b)

Fig. 3 The influence of the white noises when the prey and predator coexist in the system (2.1), where a: $b_{1}=0.7, b_{2}=0.65, c_{1}=0.15, c_{2}=0.4, \mathbf{b}: b_{1}=3.4, b_{2}=3.5, c_{1}=3, c_{2}=3.15$

For the effect of the white noise to disease spread, selecting $\sigma_{1}=0.09, \sigma_{2}=0.1$, $\omega_{1}=0.02$ and $\omega_{2}=0.04$, the other parameters are the same as those in Table 2 . Figure 4a shows that the relatively small white noise make the solutions of system (2.1) fluctuating in a large scale. Figure $4 \mathrm{~b}$ illustrates that large enough white noise leads diseases in predator population to be extinct while it is persistent in the deterministic system, which is consistent with the theoretical results of Theorem 5.2.

\section{Discussion}

In this paper, a predator-prey system with diseases in predator population only incorporating stochastic perturbations, patchy structure and transport-related inflection has been proposed and investigated. By constructing different Lyapunov functions, we proved that the existence and uniqueness of the global positive solution, stochastically ultimate boundedness of the solution and extinction of diseases of system (2.1). Compared with some published researches, ecological epidemics and white noise are incorporated in our model since infectious diseases and environmental noises are widely exist in natural world, and paly an important effect on population's evolution. Furthermore, the existing evidences show that infectious diseases will be endemic while environmental noises are ignored. However, our study shows that environmental noises are very important factors to control infectious diseases, and infectious diseases will be extinct under a certain strength of environmental noises, which is disagreement with some published works.

Moreover, the outbreak of COVID-19 shows that the transport-related inflection plays an importantly positive role in the spread of infectious diseases, and the transport-related inflection make diseases to be transmitted in a more large scale. Our investigation will also provide a strong evidence for the positive effect of the transportrelated inflection on the transmission of infectious diseases. Based on this issue, our results are agreement with previous works in this filed. Some previous works sug- 


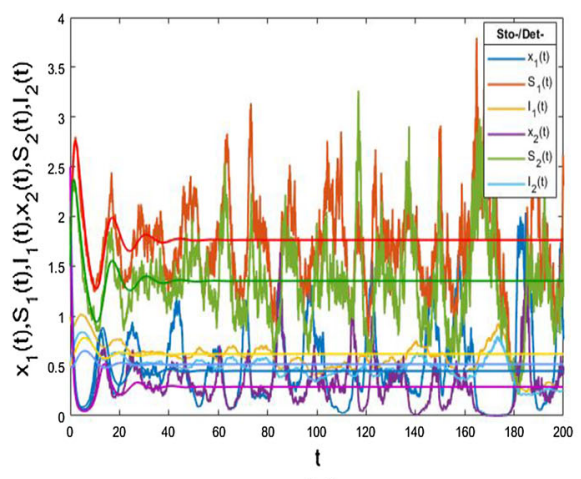

(a)

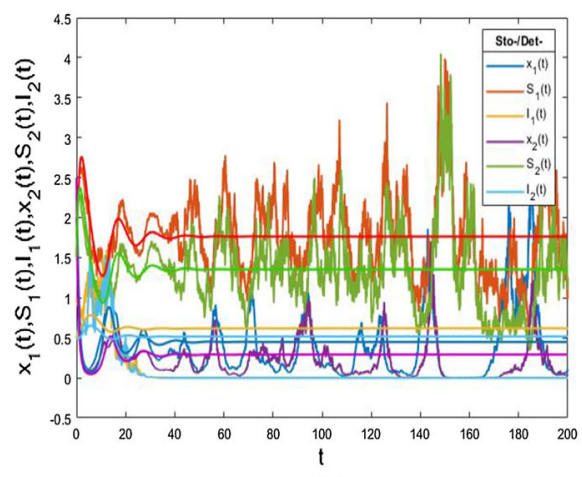

(b)

Fig. 4 The influence of the white noises when the disease exist in the system (2.1), where $\mathbf{a}: c_{1}=0.03, c_{2}=$ $0.04, \eta_{1}=0.02, \eta_{2}=0.014$, b: $c_{1}=3.15, c_{2}=3.3, \eta_{1}=4, \eta_{2}=3.75$

gested that the population's transport among different regions should be completely prohibited while infectious diseases outbroke. However, our research suggests that the transport should be prohibited under a certain scale while infectious diseases spread, and the corresponding threshold value is obtained. This controlling measure for infectious diseases could reduce economic and other losses of countries and cities as far as possible. Based on this issue, our conclusions are disagreement with some previous researches.

Furthermore, compared with stochastic models which has presented by previous works (Hu et al. 2019; Xin and Liu 2019), few of them incorporated the patchy structure into corresponding models. In fact, habitat on which populations survive are fragmented as human activities. The environmental fragmentation is negative to the population's permanence and coexistence, and will cause the loss of species biodiversity. In this paper, the patchy structure is incorporated into a stochastic ecological epidemic model, and two patches are connected by population's migrations. Hence, our model is more realistic compared with some previous researches. Meanwhile, the coexistence threshold values of interacting populations and the extinct threshold values of infectious diseases are found respectively. Our results show that the population's permanence and the disease extinction will be achieved for populations or species which survive in more complex environment. Based on this issue, our study is more useful for the biodiversity conservation and the diseases control.

Although our investigation considered many realistic issues and obtained some reasonable results, There are existing some interesting issues to be investigated furtherly. On the one hand, it is necessary to incorporate the time-delay which measures the length of the time to complete one-way transport between two patches. On the other hand, one may propose some more realistic model systems, such as considering the nonlinear incidence rate and the generalized functional response. These will be considered in future work. 
Funding This work was supported by Natural Science Foundation of Gansu Province (No. 21JR7RA165, 20JR5RA238, 21JR7RA535) and the Fundamental Research Funds for the Central Universities (lzujbky2021-54).

\section{References}

Abrams PA, Cressman R, Krivan V (2007) The role of behavioral dynamics in determining the patch distributions of interacting species. Am Nat 169:505-518

Allen LJS (2011) An introduction to stochastic processes with applications to biology, 2nd edn. CRC Press, FL, Boca Raton

Allen LJS, Bokil VA (2012) Stochastic models for competing species with a shared pathogen. Math Biosci Eng 9:461-485

Anderson RM, May RM (1986) The invasion, persistence, and spread of iufectious diseases within animal and plant communites. Phil Trans R Soc London B 314:533-570

Arifah B, Mao X (2004) Stochastic delay Lotka-Volterra model. J Math Anal Appl 292:364-380

Bao J, Shao J (2015) Permanence and extinction of regime-switching predator-prey models. SIAM J Math Anal 48:725-739

Bhattacharyya R, Mukhopadhyay B (2010) On an eco-epidemiological model with prey harvesting and predator switching: Local and global perspectives. Nonlinear Anal-Real 11:3824-3833

Cui J, Takeuchi Y, Saito Y (2006) Spreading disease with transport-related infection. J Theor Biol 239:37690

Das A, Samanta GP (2018) Stochastic prey-predator model with additional food for predator. Physica A: Stat Mech Appl 512:121-141

Fenton A, Rands SA (2006) The impact of parasite manipulation and predator foraging behaviour on predator-prey communities. Ecol 87:2832-2841

Greenhalgh D, Khan QJA, Al-Kharousi FA (2020) Eco-epidemiological model with fatal disease in the prey. Nonlinear Anal-Real 53:103072

Gulland F. M. D. (1995) The impact of infectious diseases on wild animal populations-a review, Ecology of Infectious Diseases in Natural Populations. (B. T. Grenfell and A. P. Dobson, eds). Cambridge: Cambridge University Press, 13: 20-51

Hamilton WD, May RM (1977) Dispersal in stable habitats. Nat 269:578-581

Haque, M., Venturino E. (2007) An ecoepidemiological model with disease in predator: the ratio-dependent case, Math Method Appl Sci, 30 (14)

Hethcote HW (2000) The mathematics of infectious diseases. SIAM Rev 42:599-653

Hsieh YH, Hsiao CK (2008) Predator-prey model with disease infection in both populations. Math Med Biol 25:247-266

Hu H, Xu L, Wang K (2019) A comparison of deterministic and stochastic predator-prey models with disease in the predator. Discrete Cont Dyn-B 24:2837-2863

Ji C, Jiang D (2011) Dynamics of a stochastic density dependent predator-prey system with BeddingtonDeAngelis functional response. J Math Anal Appl 381:441-453

Li X, Mao X (2009) Population dynamical behavior of non-autonomous Lotka-Volterra competitive system with random perturbation. Discret Con Dyn Syst 24:523-593

Liu X, Takeuchi Y (2006) Spread of disease with transport-related infection and entry screening. J Theor Biol 242:517-28

Liu M, He X, Yu J (2018) Dynamics of a stochastic regime-switching predator-prey model with harvesting and distributed delays. Nonlinear Anal Hybri 28:87-104

Liu M, Du C, Deng M (2018) Persistence and extinction of a modified Leslie-Gower Holling-type II stochastic predator-prey model with impulsive toxicant input in polluted environments. Nonlinear Anal Hybri 27:177-190

Mainul H (2010) A predator-prey model with disease in the predator species only. Nonlinear Anal RWA 11:2224-2236

Mao X (2007) Stochastic differential equations and applications. Horwood Publishing Limited, Chichester

Mondal S, Samanta G (2021) Time-delayed predator-prey interaction with the benefit of antipredation response in presence of refuge. Zeitschriftfr Naturforschung A 76(1):23-42 
Rebelo C, Soresina C (2020) Coexistence in seasonally varying predator-prey systems with Allee effect. Nonlinear Anal-Real 55:103140

Rudnicki R (2003) Long-time behaviour of a stochastic prey-predator model. Stoch Proc Appl 108:93-107

Saha S, Samanta GP (2019) Influence of dispersal and strong Allee effect on a two-patch predator-prey model. Int J Dyna Control 7:1321-1349

Saha S, Samanta GP (2019) Analysis of a predator-prey model with herd behavior and disease in prey incorporating prey refuge. Int J Biomath 12:133-174

Saha S, Samanta GP (2020) A Prey-predator system with disease in prey and cooperative hunting strategy in predator. J Phys A: Math Theor 53:485601

Saha S, Maiti A, Samanta GP (2018) A Michaelis-Menten Predator-prey model with strong Allee effect and disease in prey incorporating prey refuge. Int J Bifurc Chaos 28(6): 1850073

Venturino E (1993) The influence of diseases on Lotka-Volterra systems. Rocky Mount J Math 24:381-402

Venturino E (2002) Epidemics in predator-prey models: disease in the predators. Math Medic Biol 19:185205

Xin Y, Liu G (2019) Analysis of stochastic Nicholson-type delay system with patch structure. Appl Math Lett 96:223-229

Publisher's Note Springer Nature remains neutral with regard to jurisdictional claims in published maps and institutional affiliations. 